\title{
Nuclear model effects in charged-current neutrino-nucleus quasielastic scattering
}

\author{
C. Maieron, M. C. Martínez, and J. A. Caballero \\ Departamento de Física Atómica, Molecular y Nuclear, Universidad de Sevilla, Apartado Postal 1065, E-41080 Sevilla, Spain \\ J. M. Udías \\ Departamento de Física Atómica, Molecular y Nuclear, Universidad Complutense de Madrid, E-28040 Madrid, Spain
}

(Received 28 March 2003; published 8 October 2003)

\begin{abstract}
The quasielastic scattering of muon neutrinos on oxygen 16 is studied for neutrino energies between $200 \mathrm{MeV}$ and $1 \mathrm{GeV}$ using a relativistic shell model. Final state interactions are included within the distorted wave impulse approximation, by means of a relativistic optical potential, with and without imaginary part, and of a relativistic mean field potential. For comparison with experimental data the inclusive charged-current quasielastic cross section for $\nu_{\mu}-{ }^{12} \mathrm{C}$ scattering in the kinematical conditions of the LSND experiment at Los Alamos is also presented and briefly discussed.
\end{abstract}

DOI: 10.1103/PhysRevC.68.048501

PACS number(s): 25.30.Pt, 13.15.+g, 24.10.Jv

In the past few years the observation of neutrino oscillations at Super-Kamiokande [1] and the subsequent proposal and realization of new experiments, aimed at determining neutrino properties with high accuracy [2], have renovated the interest towards neutrino scattering on complex nuclei. In fact, neutrino detectors usually contain carbon or oxygen nuclei, and for a proper interpretation of the experimental results the description of the $\nu$-nucleus interaction must be accurate [3].

At intermediate neutrino energies, ranging from some hundreds $\mathrm{MeV}$ to a few $\mathrm{GeV}, \nu$-nucleus quasielastic scattering has been studied within several approaches [4]. Relativistic and nonrelativistic studies of random phase approximation have shown nuclear structure effects to be relevant only at low momentum transfers, but indications have been found that for future and precise data analyses of, e.g., atmospheric neutrino measurements, more accurate theoretical estimates may be needed. Additionally, very recently attention has been drawn towards final state interaction (FSI) effects, which, contrary to what is often assumed, may still be relevant even at the relatively high energy $E_{\nu}=1 \mathrm{GeV}$ [5].

In this contribution we study charged-current (CC) neutrino-nucleus quasielastic scattering within the framework of a relativistic shell model (RSM), already successfully employed to study exclusive electron scattering [6] and neutral current neutrino scattering [7]. We compute inclusive $\nu_{\mu^{-}}{ }^{16} \mathrm{O}$ quasielastic cross sections for three values of the incident neutrino energy, namely, $200 \mathrm{MeV}, 500 \mathrm{MeV}$, and $1 \mathrm{GeV}$, which are representative of the kinematical range where quasielastic scattering gives the main contribution to the inclusive $\nu$-nucleus process.

We describe the CC quasielastic scattering of neutrinos on a nuclear target within the impulse approximation (IA), assuming that the incident neutrino exchanges one vector boson with only one nucleon, which is then emitted, while the remaining $(A-1)$ nucleons in the target are spectators. The nuclear current is assumed to be the sum of single-nucleon currents, for which we employ the usual free nucleon expression (see Ref. [7]) with the axial form factor parametrized as a dipole with cutoff mass $M_{A}=1.026 \mathrm{GeV}$ [8], and the states of the target and residual nuclei to be adequately described by an independent particle model wave function.

To describe the bound nucleon states we use relativistic shell model wave functions, obtained as the self-consistent (Hartree) solutions of a Dirac equation, derived, within a relativistic mean field approach, from a Lagrangian containing $\sigma, \omega$, and $\rho$ mesons [9]. As the single-particle binding energies determine the threshold of the cross section for every shell, in the numerical calculations, we have used the experimental values corresponding to the binding energies of the different shells.

For the outgoing nucleon the simplest choice is to use plane wave spinors, i.e., no interaction is considered between the ejected nucleon and the residual nucleus [plane wave impulse approximation (PWIA)]. For a more realistic description, FSI effects should be taken into account. In our formalism this is done by using distorted waves which are

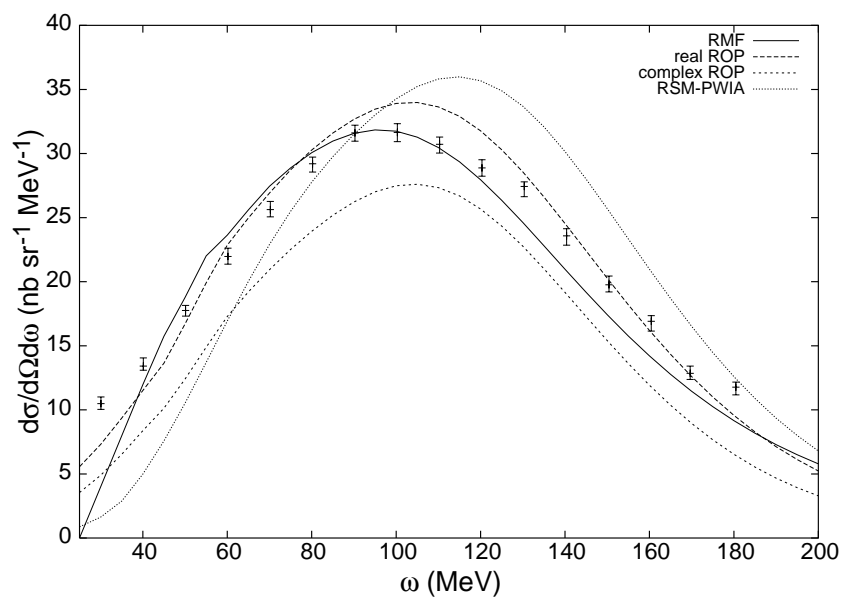

FIG. 1. Differential cross section $d \sigma / d \Omega d \omega$ vs the energy transfer for inclusive quasielastic electron scattering on ${ }^{12} \mathrm{C}$ for a momentum transfer $q \simeq 400 \mathrm{MeV} / c$. The solid curve corresponds to the RSM with FSI described by the RMF, while the long-dashed curve corresponds to the real ROP and the short-dashed curve to the complex ROP. Finally, the dotted curve does not include FSI. Experimental data are from Ref. [11]. 

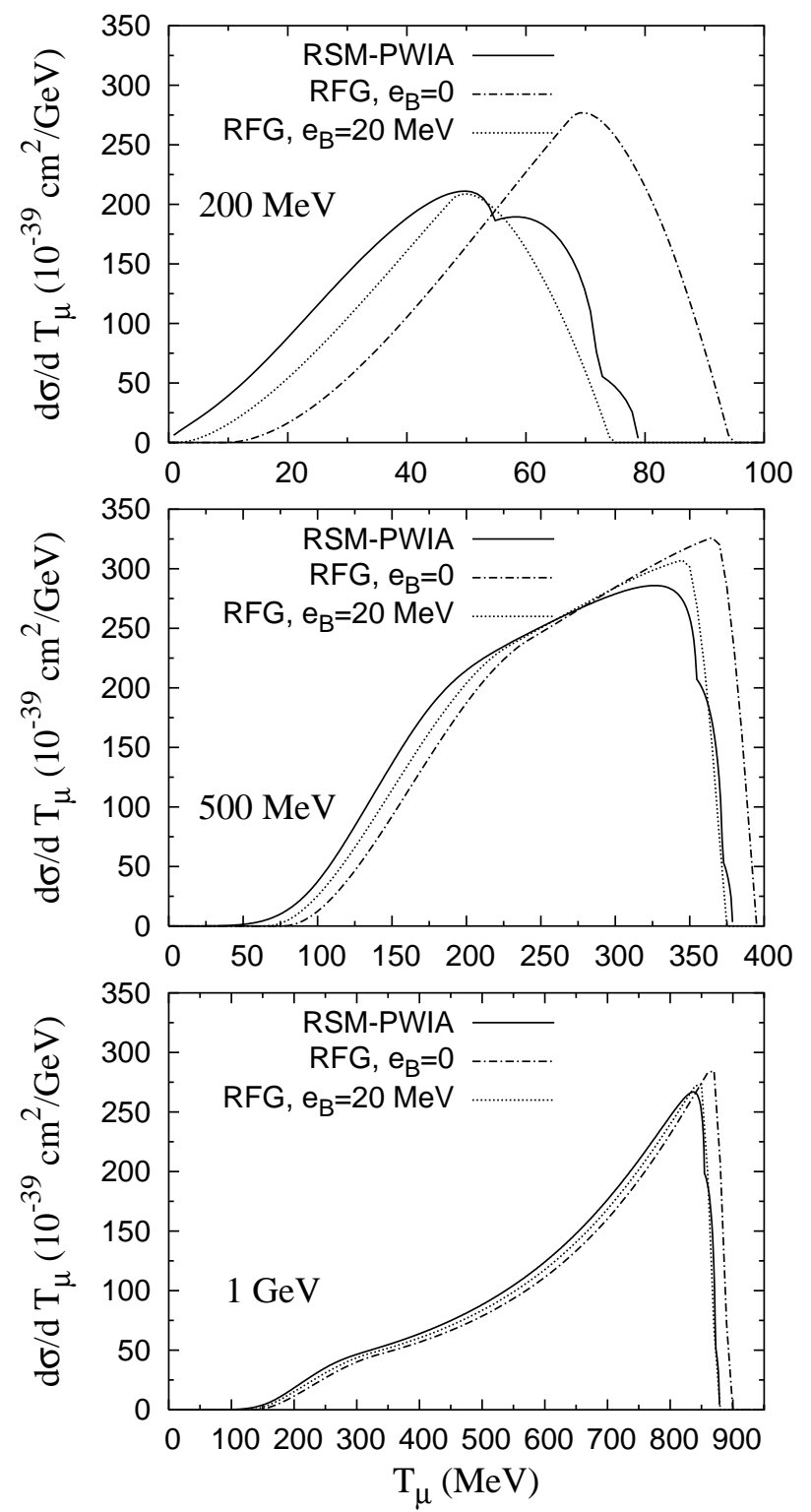

FIG. 2. Differential cross section $d \sigma / d T_{\mu}$ vs the outgoing muon kinetic energy, for the quasielastic scattering of muon neutrinos on ${ }^{16} \mathrm{O}$ and for three choices of the incident neutrino energy: $E_{\nu}$ $=200 \mathrm{MeV}$ (upper panel), $500 \mathrm{MeV}$ (middle panel), and $1 \mathrm{GeV}$ (lower panel). The solid curves correspond to the RSM with no final state interaction, while the remaining curves are calculated within the RFG, with $k_{F}=225 \mathrm{MeV}$, and $e_{B}=0$ (dot-dashed) and $e_{B}$ $=20 \mathrm{MeV}$ (dotted).

given as solutions of a Dirac equation containing a phenomenological relativistic optical potential (ROP), consisting of a real part, which describes the rescattering of the ejected nucleon and of an imaginary part that accounts for the absorption of it into unobserved channels. In this work we use the ROP corresponding to the energy-dependent-Adependent-1 (EDAD-1) single-nucleon parametrization presented in Ref. [10]. The use of this phenomenological ROP leads to an excellent agreement between theoretical calculations and data for exclusive $\left(e, e^{\prime} N\right)$ observables [6]; however, some caution should be taken in extending the conclu-
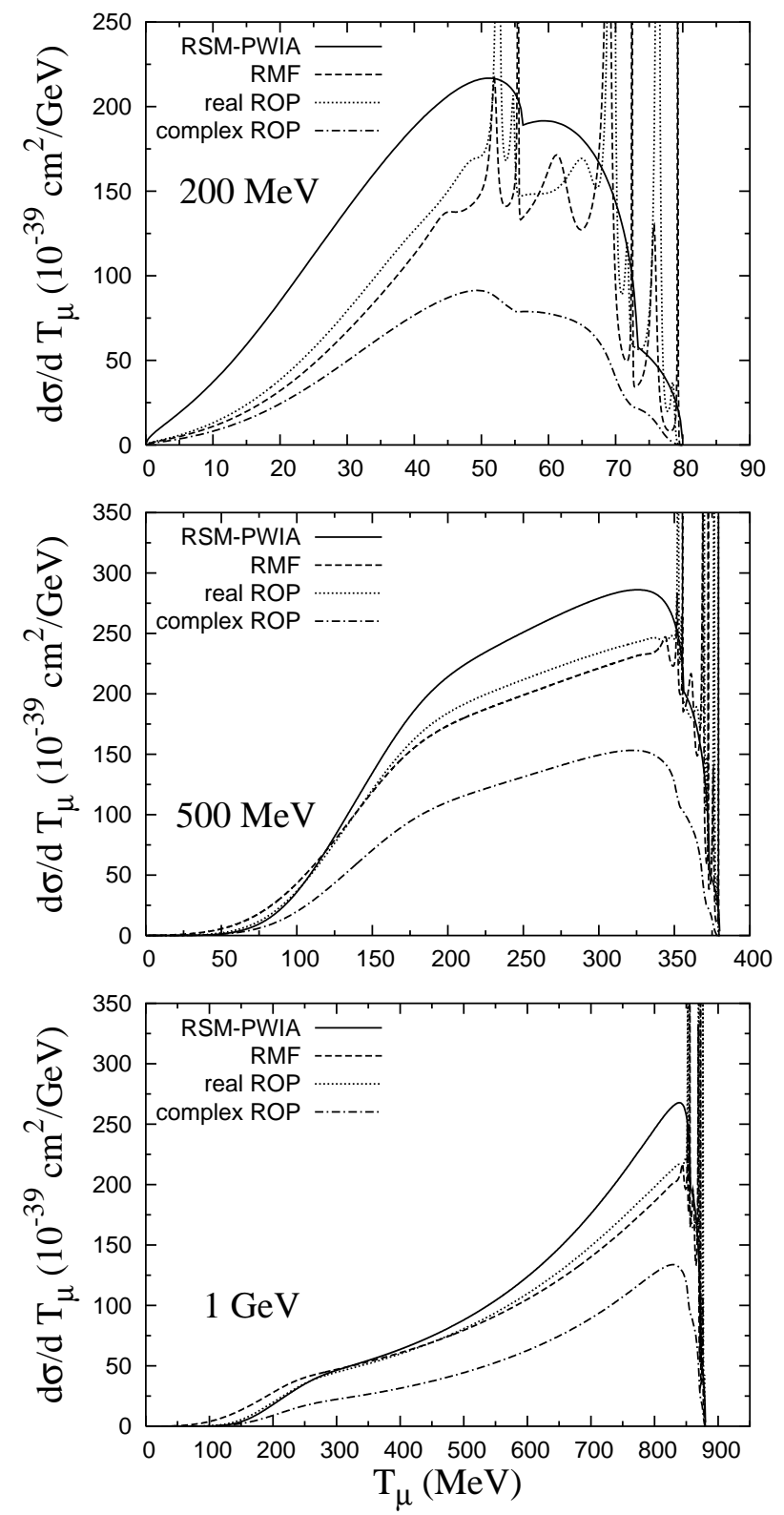

FIG. 3. Same as Fig. 2, but including FSI effects. All curves are calculated within the RSM model in PWIA (solid), and within the RMF (dashed), real ROP (dotted), and complex ROP (dot-dashed) approaches.

sions drawn from the analysis of exclusive reactions to inclusive ones. In the latter, unless a selection of the singlenucleon knockout contribution is experimentally feasible, all final channels are included and thus the imaginary term in the optical potential leads to an overestimation of FSI effects. This is clearly illustrated in Fig. 1 where we compare our theoretical results with the experimental cross section corresponding to inclusive quasielastic electron scattering on ${ }^{12} \mathrm{C}$ for a momentum transfer equal to $400 \mathrm{MeV} / c$ (similar results are obtained for 300 and $500 \mathrm{MeV} / c$ ). In our calculation besides the complex ROP, we also consider the potential obtained by setting the imaginary part of the ROP to zero. Additionally, one may also use distorted waves which are obtained as the solutions in the continuum of the same Dirac 


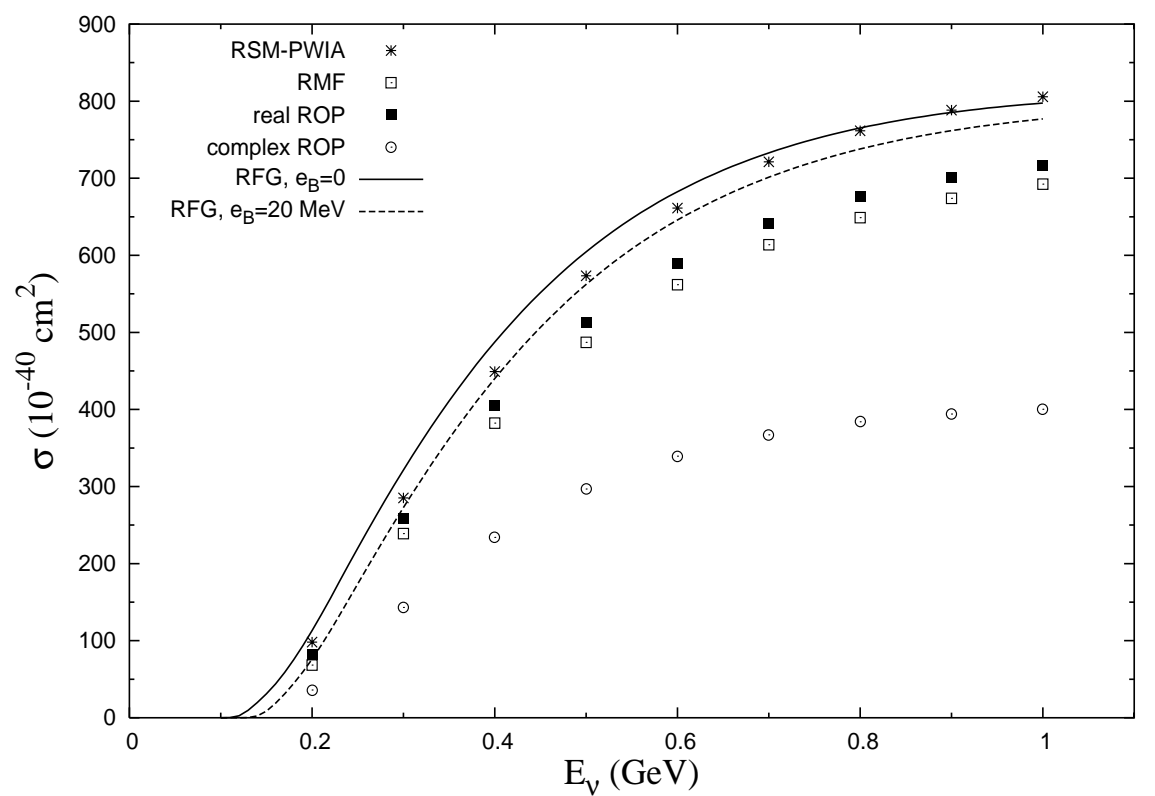

FIG. 4. Integrated cross section $\sigma\left(E_{\nu}\right)$ for the quasielastic scattering of muon neutrinos on ${ }^{16} \mathrm{O}$ as a function of the incident neutrino energy. The curves are calculated within the RFG model with $k_{F}=225 \mathrm{MeV}$, and binding energy $e_{B}=0$ (solid line) and $e_{B}$ $=20 \mathrm{MeV}$ (dashed). The points correspond to RSM calculations without FSI (stars) and with FSI effects taken into account within the RMF (empty squares), real ROP (full squares), and complex ROP (circles) approaches. equation used to describe the initial bound nucleon. We refer to this approach, which should be adequate to describe FSI at moderate energy transfer, as relativistic mean field (RMF). As shown in Fig. 1, the complex ROP results clearly underestimate the data, while the reverse occurs for the PWIA calculation. On the other hand, the RMF and purely real ROP agree much better with experiment, particularly RMF for small transfer energy and real ROP for higher $\omega$. We believe that the RMF and real ROP results indicate a reasonable "band" where FSI effects should lie.

Let us now present our results for neutrino scattering on ${ }^{16} \mathrm{O}$. To better illustrate our model, we start by neglecting FSI and comparing RSM-PWIA results with inclusive cross sections obtained within the relativistic Fermi gas (RFG) [7]. This is done in Fig. 2, which shows the differential cross section $d \sigma / d T_{\mu}$ as a function of the outgoing muon kinetic energy. With respect to the RFG curve calculated with no binding energy we observe that the RSM cross section is reduced and shifted towards lower $T_{\mu}$ values, in a way which is similar to the effect of an average binding energy in the RFG. In addition, the RSM cross section has a different shape, due to the different momentum distributions of the single-nucleon shells contributing to the process. Since the various shells have different binding energies, the corresponding contributions to the cross section go to zero at different values of $T_{\mu}$ and this gives rise to the structure of $\left(d \sigma / d T_{\mu}\right)$ observed at large $T_{\mu}$. Figure 2 shows that nuclear model effects on the cross sections can be rather large at low neutrino energy, but become less relevant as $E_{\nu}$ increases, practically disappearing at $E_{\nu}=1 \mathrm{GeV}$.

On the other hand, the behavior of FSI effects is quite different, as illustrated in Fig. 3. Here the results obtained with the RSM in PWIA are compared with the cases where FSI are described within the RMF, the real ROP and the complex ROP approaches. The use of real potentials (RMF and real ROP) for describing the final nucleon states leads to the resonant structure observed for relatively high $T_{\mu}$ (that is, small energy transfer $\omega$ ). Note that in this work we only include single-particle excitations within a mean field picture. Including residual interactions would make the width and number of resonances to be considerably larger.

We observe that FSI effects produce a reduction of the cross section, particularly important in the case of the complex ROP model due to the absorption introduced by the imaginary term: about $60 \%$ for $E_{\nu}=200 \mathrm{MeV}$ and $50 \%$ for $E_{\nu}=500 \mathrm{MeV}$ and $E_{\nu}=1 \mathrm{GeV}$ in the region close to the maximum. For the RMF and real ROP, the reduction, similar in both cases, is about $30-40 \%$ for $E_{\nu}=200 \mathrm{MeV}$ and $20 \%$ for the other energy values.

Nuclear model effects on integrated cross sections are studied in Fig. 4, where the cross section $\sigma\left(E_{\nu}\right)$ is plotted as a function of the incident neutrino energy. Here the contributions coming from the RMF and ROP resonances have been included in the calculation, in order to respect the completeness of the set of final states predicted by the model. These contributions are important at $E_{\nu}=200 \mathrm{MeV}$, where they amount to about $10 \%$ of the integrated cross section, while at higher energies these effects are about $2 \%$ $(500 \mathrm{MeV})$ and $1 \%(1 \mathrm{GeV})$. Again we see that within the PWIA the discrepancy between different nuclear models is relatively small and decreases with increasing neutrino energy. On the contrary FSI effects remain sizable even at large $E_{\nu}$. As in the previous figure, the imaginary term in the ROP leads to a too large reduction $(\sim 50 \%)$ of the integrated cross section. The results for the RMF and real ROP models, which are more reliable, show a smaller, but still sizable $\left(\sim 15 \%\right.$ at $\left.E_{\nu}=1 \mathrm{GeV}\right)$ reduction.

The results shown in Figs. 3 and 4 lead us to conclude that FSI effects should be carefully considered in neutrino experiments which use oxygen based detectors. Analogous conclusions can be drawn for the case of a ${ }^{12} \mathrm{C}$ target.

For the purpose of comparison with the experiment, let us consider the inclusive ${ }^{12} \mathrm{C}\left(\nu_{\mu}, \mu^{-}\right) X$ cross section measured by the LSND Collaboration at Los Alamos, using a piondecay-in-flight $\nu_{\mu}$ beam, with energies ranging from muon threshold to $300 \mathrm{MeV}$, and a large liquid scintillator detector 


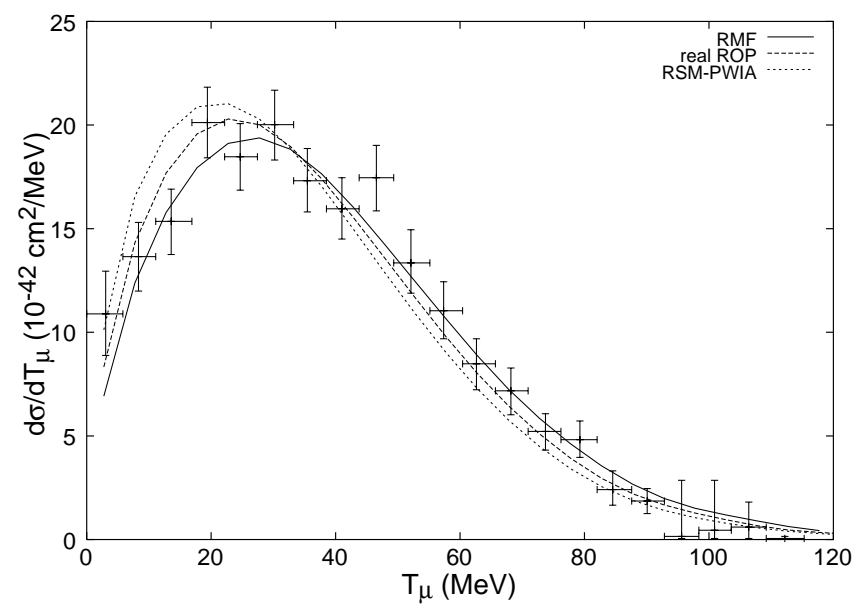

FIG. 5. Observed distribution of muon kinetic energies $T_{\mu}$ compared with the flux-averaged predictions of our RSM, in PWIA (dotted line) and including FSI within the RMF (solid) and purely real ROP (dashed) frameworks. The theoretical distributions have been normalized to give the same integrated values as the experimental points, and have been folded in energy with a bin size of 5 $\mathrm{MeV}$, the same employed for the experimental data. Data are from Albert et al. [12].

[12]. This experiment has been compared to very different theoretical approaches [13], but an important discrepancy still remains. Although at these low energies processes different from the quasielastic nucleon knockout affect the in- clusive cross section, we consider our FSI approach to be useful to estimate integrated cross sections.

In Fig. 5 we show the observed [12] and calculated distribution of events, averaged over the 1994 Los Alamos neutrino spectrum $\phi\left(E_{\nu}\right)$ within the energy range $E_{\nu}$ $=123.1-300 \mathrm{MeV}$. The shape and position of the maximum of the experimental distribution are approximately reproduced by the three calculations, but the results that include FSI with the RMF potential are clearly favored by the data. This is consistent with the fact that including FSI with the mean field potential should be adequate at moderate kinetic energy of the ejected nucleon. However, the values we obtain for the flux-averaged integrated cross section overestimate the measured cross section by $\approx 50 \%$. More precisely, in the RSM, we obtain $\langle\sigma\rangle=20.5$ (PWIA), 16.8 (RMF), and 15.1 (real ROP) $10^{-40} \mathrm{~cm}^{2}$. Additional corrections due to the outgoing muon Coulomb distortion, evaluated within the effective momentum approximation [14], further increase these numbers by $5-10 \%$. The corresponding final measured experimental value is $(10.6 \pm 0.3 \pm 1.8) \times 10^{-40} \mathrm{~cm}^{2}$ [12]

This work was partially supported by DGI (Spain) under Contract Nos. BFM2002-03315, FPA2002-04181-C04-04, and BFM2000-0600 and by the Junta de Andalucía. C.M. acknowledges MEC (Spain) for a postdoctoral stay at University of Sevilla (Contract No. SB2000-0427). M.C.M. acknowledges support from the Fundación Cámara (University of Sevilla).
[1] Y. Fukuda et al., Super-Kamiokande Collaboration, Phys. Rev. Lett. 81, 1562 (1998); C. K. Jung, C. McGrew, T. Kajita, and T. Mann, Annu. Rev. Nucl. Part. Sci. 51, 451 (2001).

[2] See, for example, A. De Santo, Int. J. Mod. Phys. A 16, 4085 (2001).

[3] See Neutrino Nucleus Interactions in the Few Gev Region, Proceedings of the First International Workshop, Nuint01, Tsukuba, Japan, 2001, edited by J. G. Morfin, M. Sakuda, and Y. Suzuki (North-Holland, Amsterdam, 2002).

[4] T. K. Gaisser and J. S. O'Connell, Phys. Rev. D 34, 822 (1986); H. c. Kim, J. Piekarewicz, and C. J. Horowitz, Phys. Rev. C 51, 2739 (1995); H. c. Kim, S. Schramm, and C. J. Horowitz, ibid. 53, 2468 (1996); 53, 3131 (1996); S. K. Singh and E. Oset, ibid. 48, 1246 (1993); J. Engel, E. Kolbe, K. Langanke, and P. Vogel, Phys. Rev. D 48, 3048 (1993); J. Marteau, J. Delorme, and M. Ericson, Nucl. Instrum. Methods Phys. Res. A 451, 76 (2000).

[5] C. Bleve et al., Astropart. Phys. 16, 145 (2001); G. Cò, C. Bleve, I. De Mitri, and D. Martello, Nucl. Phys. B, Proc. Suppl. 112, 210 (2002).

[6] J. M. Udías et al., Phys. Rev. C 48, 2731 (1993); 51, 3246
(1995); 53, R1488 (1996); 64, 024614 (2001).

[7] W. M. Alberico et al., Nucl. Phys. A623, 471 (1997).

[8] V. Bernard, L. Elouadrhiri, and U. G. Meissner, J. Phys. G 28, R1 (2002).

[9] C. J. Horowitz and B. D. Serot, Nucl. Phys. A368, 503 (1981); Phys. Lett. 86B, 146 (1979); B. D. Serot and J. D. Walecka, Adv. Nucl. Phys. 16, 1 (1986).

[10] E. D. Cooper, S. Hama, B. C. Clark, and R. L. Mercer, Phys. Rev. C 47, 297 (1993).

[11] P. Barreau et al., Nucl. Phys. A402, 515 (1983).

[12] M. Albert et al., LSND Collaboration, Phys. Rev. C 51, 1065 (1995); C. Athanassopoulos et al., LSND Collaboration, ibid. 56, 2806 (1997); L. B. Auerbach et al., LSND Collaboration, ibid. 66, 015501 (2002).

[13] See N. Jachowicz, K. Heyde, J. Ryckebusch, and S. Rombouts, Phys. Rev. C 65, 025501 (2002); C. Volpe et al., ibid. 62, 015501 (2000); E. Kolbe et al., ibid. 52, 3437 (1995); Nucl. Phys. A652, 91 (1999); Y. Umino, J. M. Udías, and P. J. Mulders, Phys. Rev. Lett. 74, 4993 (1995); Y. Umino and J. M. Udías, Phys. Rev. C 52, 3399 (1995).

[14] C. Giusti and F. D. Pacati, Nucl. Phys. A473, 717 (1987), and references therein. 